\title{
WATER ADDITION TO WAMUS DIRTY GAS SYSTEM.
}

\section{ENGINEERING NOTE}

3823.134-EN-581

February 16, 2006

Author: Mike Sarychev 


\section{Water addition to WAMUS Dirty gas system.}

Total flow through the whole WAMUS Dirty system $-135 \mathrm{l} / \mathrm{min}$;

Currently, $\mathrm{H}_{2} \mathrm{O}$ content is $600 \mathrm{ppm}(0.45 \mathrm{mg} / \mathrm{l})$.

In order to rise $\mathrm{H}_{2} \mathrm{O}$ content to $1500 \mathrm{ppm}(1.2 \mathrm{mg} / \mathrm{l})$, we need to add $0.75 \mathrm{mg} / \mathrm{l}$.

In order to rise $\mathrm{H}_{2} \mathrm{O}$ content to $2500 \mathrm{ppm}(1.8 \mathrm{mg} / \mathrm{l})$, we need to add $1.35 \mathrm{mg} / \mathrm{l}$.

Gas flowing through a bubbler filled with water at room temperature $-23 \mathrm{C}$ (saturated) has water content of $23 \mathrm{mg} / \mathrm{l}$, therefore, flow through a bubbler has to be $4.4 \mathrm{l} / \mathrm{min}$ (to get $1500 \mathrm{ppm}$ ); or $7.9 \mathrm{l} / \mathrm{min}$ (to get $2500 \mathrm{ppm}$ ).

WAMUS Dirty system volume is $240 \mathrm{~m}^{3}$. It will need $180 \mathrm{~g}$ to increase $\mathrm{H}_{2} \mathrm{O}$ content to 1500 ppm or $325 \mathrm{~g}$ to go to $2500 \mathrm{ppm}$. It will take 1.25 days (or 2.25 days to go to $2500 \mathrm{ppm}$ with 4.4 $1 /$ min flow.

Steady state flow through a bubbler will be $0.5 \mathrm{l} / \mathrm{min}$ to maintain $1500 \mathrm{ppm}$ and $0.8 \mathrm{l} / \mathrm{min}$ to maintain $2500 \mathrm{ppm}$. Water usage will be around $17.5 \mathrm{ml}$ per 24 hours for $1500 \mathrm{ppm}$; or around $26 \mathrm{ml}$ per 24 hours for $2500 \mathrm{ppm}$.

Proposed flow diagram:

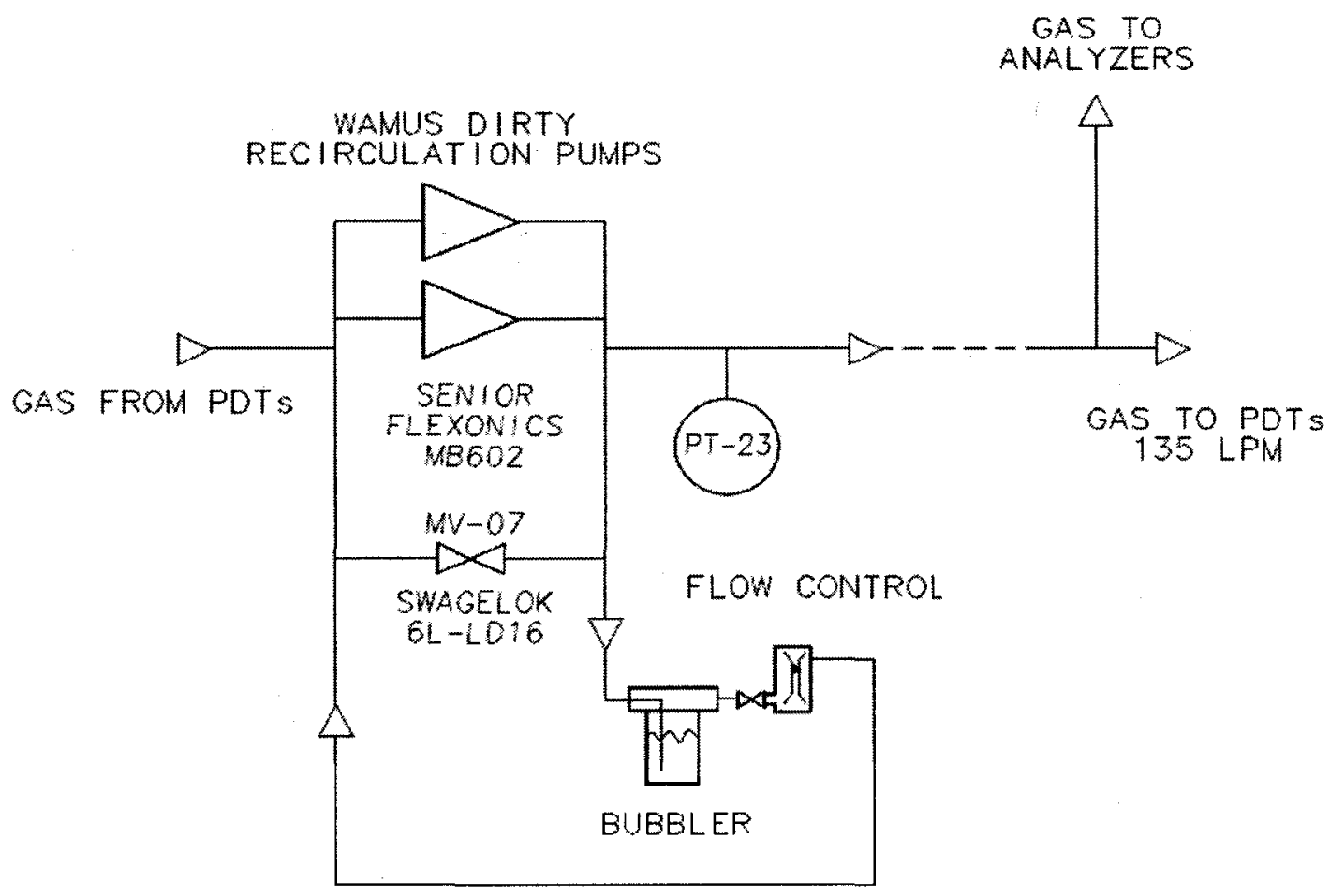

A bubbler and a flow controller (0-5 lpm or 0-10 scfh) can be connected in parallel with MV-07 pumps bypass valve in room 510 (we have available ports in existing piping to do it). Existing analyzers will be used to check $\mathrm{H}_{2} \mathrm{O}$ content in gas. 


\section{Water addition to a single PDT test.}

Total flow through the whole WAMUS Dirty system - $1351 / \mathrm{min}$;

72 PDT in the system;

Estimated flow through a single WAMUS Dirty system PDT $-1.8751 / \mathrm{min}$.

Currently, $\mathrm{H}_{2} \mathrm{O}$ content is $600 \mathrm{ppm}(0.45 \mathrm{mg} / \mathrm{l})$; need to rise to $2500 \mathrm{ppm}(1.85 \mathrm{mg} / \mathrm{l})$ - add $1.4 \mathrm{mg} / \mathrm{l}$.

Gas flowing through a bubbler filled with water at room temperature $-22 \mathrm{C}$ (saturated) has water content of $19 \mathrm{mg} / \mathrm{l}$, therefore flow through a bubbler has to be $0.141 / \mathrm{min}$ or $0.29 \mathrm{ft}^{3} / \mathrm{hr}$. Water usage will be around $4 \mathrm{ml}$ per 24 hours.

Proposed flow diagram:

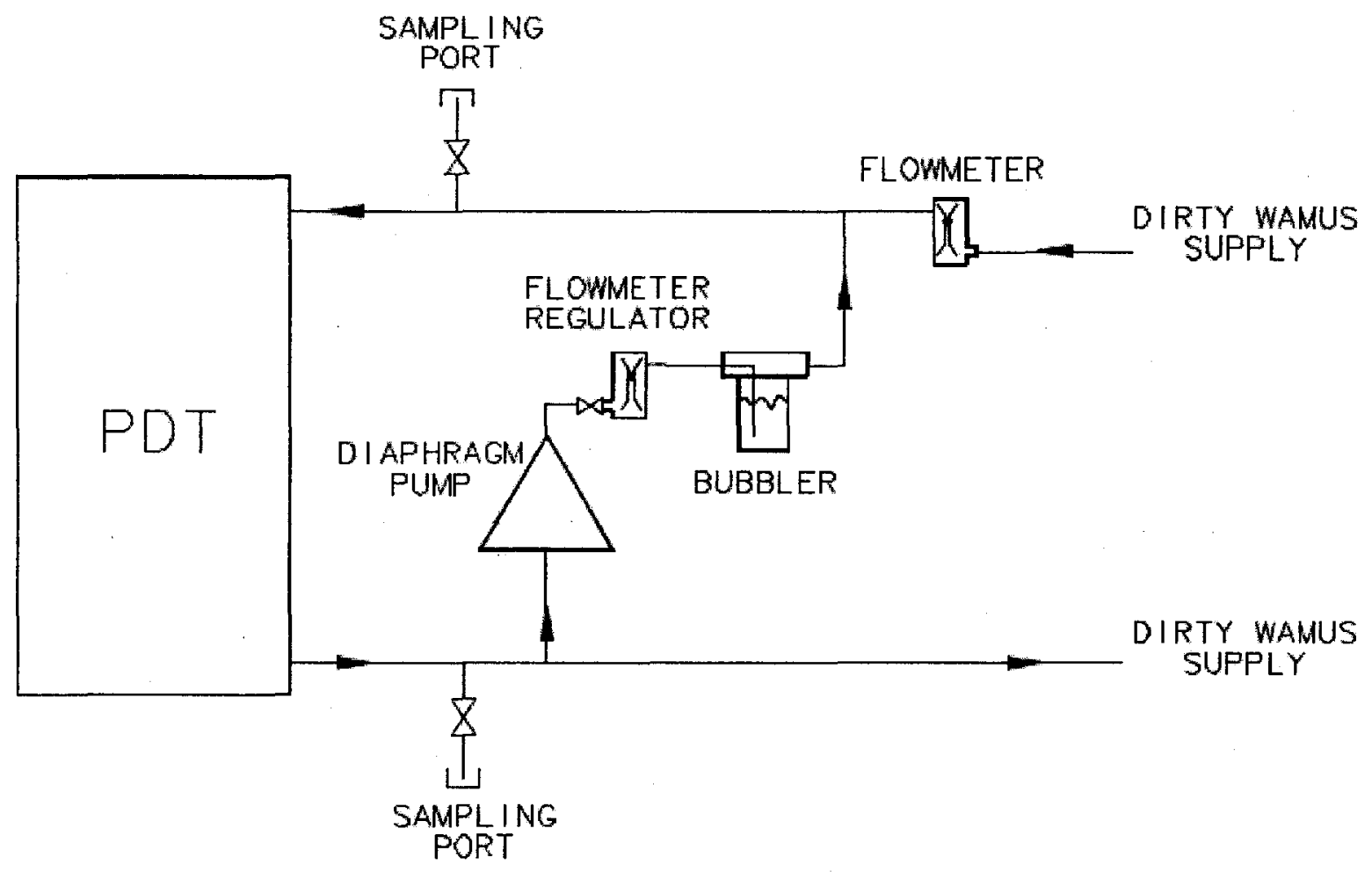

SHAW hygrometer might be used to monitor water content. $2500 \mathrm{ppm}$ correspond to $-10 \mathrm{C}$ Dew point.

PDT to be tested is one of four suggested by Penny Kasper - 123, 207, 230 or 133 - will be chosen after Collision Hall access and site inspection (the easiest one to reach and access to in-out ports, power outlet nearby, probably a TV camera to watch hygrometer readings). 


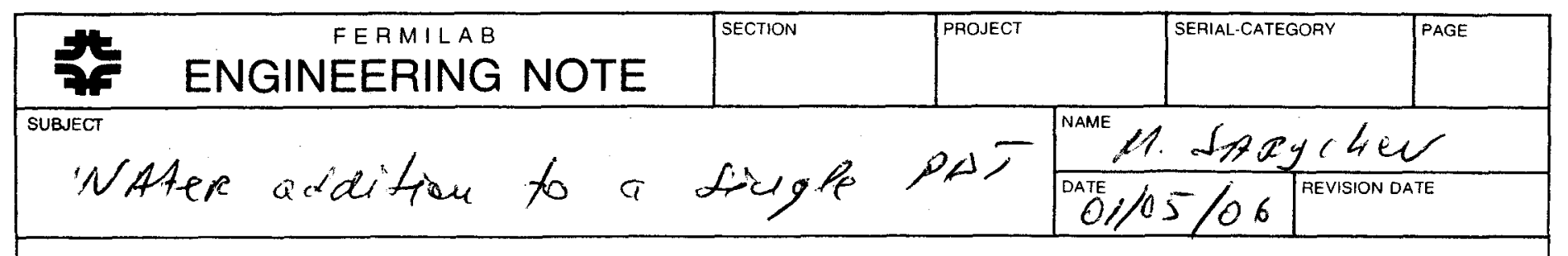

Curvently 600 ppar $\rightarrow 0.45 \mathrm{mg} / \mathrm{c}$

needs $2500 \mathrm{ppm} \rightarrow 1.85 \mathrm{mg} / \mathrm{c}$

$$
\Rightarrow \text { add } 1.4 \mathrm{ag} / \mathrm{c}
$$

$$
22^{\circ} \mathrm{C} \mathrm{D.P.} \rightarrow H_{2} O_{\text {content }}=19 \mathrm{ag} / \mathrm{c}
$$

Average flow ther a divgle PDI

$$
135 / 72=1.875 \text { l/ain }
$$

flow aeeded: $1.4 \mathrm{mg} / \mathrm{e} \times 1.875 / \mathrm{min}=2.625 \mathrm{mg} / \mathrm{min}$

$$
Q: \frac{2.625 \mathrm{mg} / \mathrm{min}}{19 \mathrm{mg} / \mathrm{l}}\left[\begin{array}{l}
=0.14 \mathrm{l} / \mathrm{aria} \\
=0.29 \mathrm{ft}^{3} / \mathrm{HR}
\end{array}\right.
$$

$W_{\text {ater usege }} 2.625 \mathrm{mg} / \mathrm{min}=157.5 \mathrm{mg} / \mathrm{he}^{2}=3.78 \mathrm{~g} / \mathrm{sar}$

$$
(1 \text { teaspon }=5 \mathrm{~g})
$$

Flowmeter zeadiugs:

$$
Q=\frac{\text { SCAMAiR }}{f} \quad f=\sqrt{\text { Spec.gzavity }}
$$

$$
\begin{gathered}
S_{G}: C H_{Y}-0.55, \quad C F_{4}=3.038 \text { AR }=1.38 \\
S_{G_{\text {MiN }}}=0.08 \cdot 0.55+0.08 \cdot 3.038+0.84 \cdot 1.38=1.45 \\
f=1.2 \quad \text { SCFH BiC requind }=0.29 \cdot 1.2=0.35544
\end{gathered}
$$




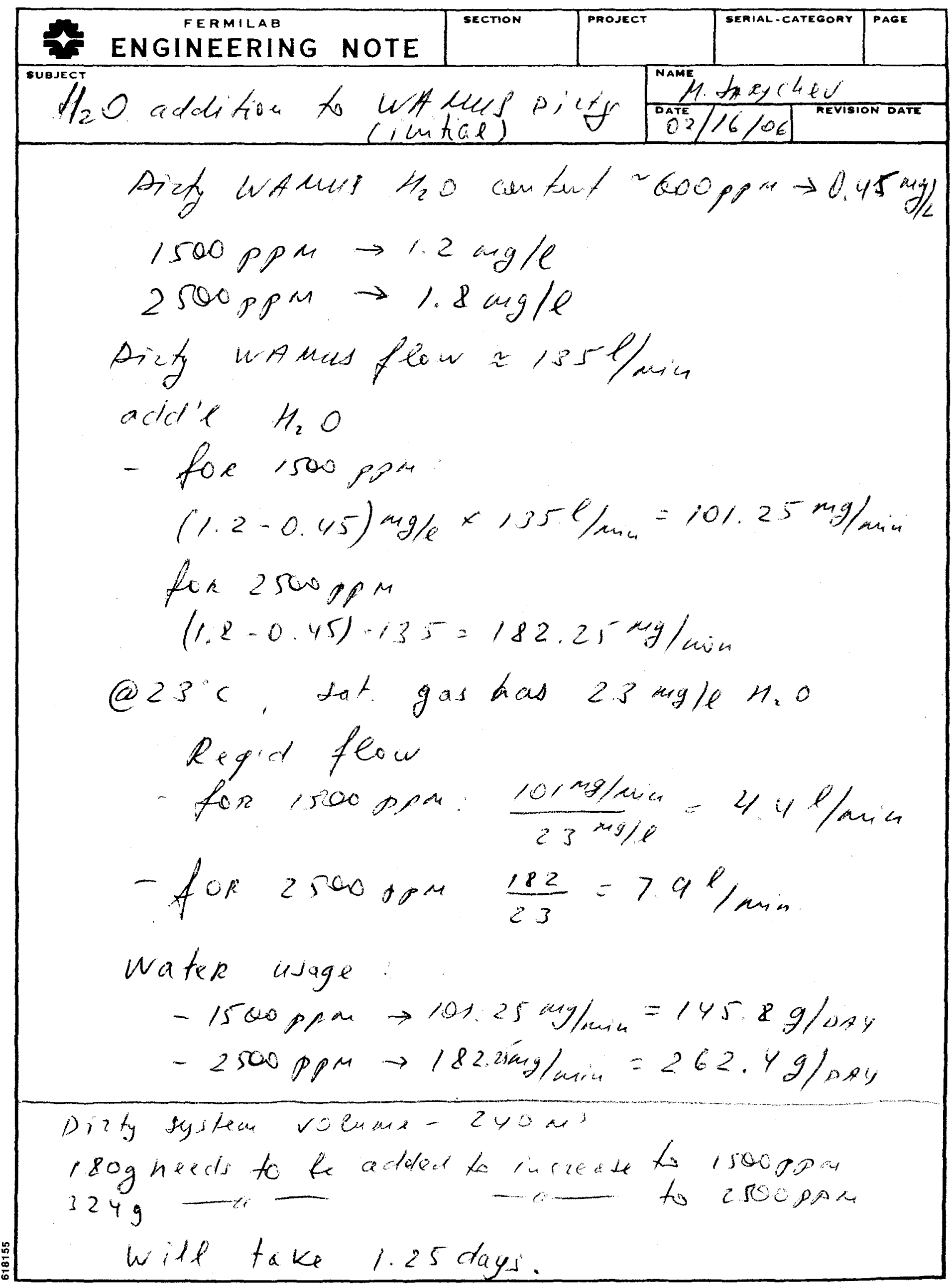


Water usage

$$
\begin{aligned}
& 1500 \text { ppm - } 17.3 \mathrm{~g} / \mathrm{dag} \\
& 2.300 \mathrm{pgm}-26 \mathrm{~g} / \mathrm{day}
\end{aligned}
$$

